\title{
Pädagogische Interaktionen als Grundbaustein der Lehrperson-Schüler*innen-Beziehung. Die Erfassung mit State Space Grids
}

\author{
Marion Scherzinger $(\mathbb{D} \cdot$ Benjamin Roth $\cdot$ Alexander Wettstein
}

Eingegangen: 31. Januar 2020 / Überarbeitet: 29. September 2020 / Angenommen: 26. Oktober 2020 /

Online publiziert: 13. November 2020

(C) Der/die Autor(en) 2020

Zusammenfassung Die Lehrperson-Schüler*innen-Beziehung ist eine wichtige Grundlage für Lehr-Lern-Prozesse. Die vorliegende Studie geht der Frage nach, inwieweit die globale Einschätzung der LS-Beziehung (Makroebene) mit den Interaktionen zwischen Lehrperson und Schüler*innen im Unterricht (Mikroebene) von 5. und 6. Primarschulklassen in der Schweiz zusammenhängen. Dazu wurden videografierte Unterrichtsstunden von 16 Lehrpersonen mit State Space Grids (SSG) analysiert. Diese Methode ermöglicht es, Interaktionen im Unterricht grafisch darzustellen und auszuwerten. Die mikrogenetischen Ergebnisse wurden anschließend mit den Fragebogeneinschätzungen der LS-Beziehung aus Sicht der Schüler*innen und der Lehrpersonen korreliert. Die Ergebnisse der Korrelationsanalysen zeigen, dass die globale Beziehungseinschätzung der Schüler*innen stärker mit den im Unterricht ablaufenden Interaktionen zusammenhängt als die der Lehrpersonen. Eine negative Beziehungseinschätzung (Fragebogen) korreliert mit beobachtetem gegenseitig unfreundlichem Verhalten im Unterricht und einer hohen Variabilität der unterrichtlichen Interaktionen (Videobeobachtung). Die Erkenntnisse liefern Anregungen zur Reflexion der LS-Beziehung in der Aus- und Weiterbildung von Lehrpersonen.

Schlüsselwörter Lehrperson-Schüler*innen-Beziehung · Unterricht ·

Klassenführung · Soziale Interaktionen · State Space Grids

M. Scherzinger $(\bowtie) \cdot$ B. Roth $\cdot$ A. Wettstein

Institut für Forschung, Entwicklung und Evaluation, Pädagogische Hochschule Bern, Fabrikstrasse

2a, 3012 Bern, Schweiz

E-Mail: marion.scherzinger@phbern.ch

B. Roth

E-Mail: benjamin.roth@nms.phbern.ch

A. Wettstein

E-Mail: alexander.wettstein@phbern.ch 


\title{
Pedagogical interactions as foundation of the teacher-student relationship. The assessment with State Space Grids
}

\begin{abstract}
A good teacher-student relationship is fundamental to teaching and learning. The present study explores the association between the perceived teacher-student relationship and the ongoing teacher-student interactions in 5th and 6th grade Swiss classrooms. We videotaped sixteen lessons and evaluated them by employing State Space Grids (SSG), a dynamic system research method that makes possible mapping real-time interactions in systems. We determined the relationship between the observed interactions (micro-level) and ratings of teacher-student relationships (macrolevel) by correlational analysis. The results show a stronger association between the students' view of the teacher-student relationship and observable interactions in the classroom compared to the teachers' view. A negative perception of the teacher-student relationship is associated with interactions with mutually low communion and higher variability of the teacher-student interactions in the classroom. The research findings provide suggestions to reflect on teacher-student relationships to be taken into account in teacher education and training.
\end{abstract}

Keywords Teacher-student relationship - Classroom - Classroom management · Pedagogical interactions $\cdot$ State space grids

\section{Einleitung}

Der Beziehung zwischen Lehrperson und Schüler*innen (LS-Beziehung) kommt eine Schlüsselrolle für das Lernen und Lehren $\mathrm{zu}$, sie bildet nebst einem qualitativ guten, kognitiv aktivierenden und geführten Unterricht eine wichtige Grundlage für erfolgreiche Lehr-Lern-Prozesse (Pianta et al. 2003; Wentzel 2012). In der Unterrichtsforschung werden unterschiedliche Dimensionen eines qualitativ guten Unterrichts unterschieden (vgl. Einsiedler 2017; Ophardt und Thiel 2017; Praetorius et al. 2018). Bei Klieme et al. (2006) sind Klassenführung, unterstützendes Unterrichtsklima und kognitive Aktivierung zentrale Qualitätsdimensionen und auch Pianta und Hamre (2009) gehen von ähnlichen Dimensionen aus: emotional support, classroom organization und instructional support. Die Bedeutung einer wertschätzenden und vertrauensvollen LS-Beziehung zeigt sich im unterstützenden Unterrichtsklima bzw. der emotionalen Unterstützung (emotional support). Dazu gehört die konstruktive, emotionale und soziale Unterstützung der Schüler*innen durch die Lehrperson, welche für das Engagement und die Motivation der Lernenden förderlich ist. Inwiefern Schüler*innen sozial unterstützt werden und sozial eingebunden sind, zeigt sich u.a. auf der Beziehungsebene bzw. in den pädagogischen Interaktionen im Unterricht. Untersuchungen haben ergeben, dass gute LS-Beziehungen, die sich durch Nähe, Sicherheit, Vertrauen und Unterstützung auszeichnen (Wentzel 2012), positiv mit der Lernmotivation (Wentzel 2010), den schulischen Leistungen (Pianta et al. 2003) und der psychosozialen Entwicklung von Schüler*innen (Davis 2003; Pianta 2006; Obsuth et al. 2017; Wentzel 2002) zusammenhängen wie auch positiv mit der Job- 
zufriedenheit und negativ mit Burnout von Lehrpersonen korrelieren (Chang 2009; Friedman 2006; Spilt et al. 2011).

LS-Beziehungen entstehen durch wiederholte Interaktionen im Unterricht (Hinde 1976a, 1976b; Hinde und Tajfel 1979) und die wahrgenommene Beziehung beeinflusst wiederum das zwischenmenschliche Verhalten (Pianta et al. 2003). In vielen Studien wird die LS-Beziehung meist global durch die Befragung von Lehrpersonen und/oder von Schüler*innen erfasst (z. B. ,Ich mag meine Lehrperson“). Dabei zeigt sich eine schwache Übereinstimmung zwischen den Einschätzungen der Lehrpersonen und der Schüler*innen (Wettstein, Ramseier et al. 2016). Schüler*innen schätzen die LS-Beziehung positiver ein, wenn die Lehrperson versucht, eine emotionale Bindung zu ihnen aufzubauen und sich fair verhält (Suldo et al. 2009). Bislang kaum erforscht wurde die Frage, inwiefern die globale Beziehungseinschätzung mit konkreten, im Unterricht beobachtbaren Interaktionen zusammenhängt. Studien aus den Niederlanden untersuchten etwa den Zusammenhang zwischen der Beziehungseinschätzung der Schüler*innen mit dem beobachteten Verhalten der Lehrpersonen. So hängen etwa Bestrafen, Anschreien oder sarkastische Bemerkungen der Lehrpersonen negativ mit der LS-Beziehung aus Schüler*innensicht zusammen (Mainhard et al. 2011). In einer anderen Studie analysierten Mainhard et al. (2012) an zwei Schulklassen zusätzlich zum Verhalten der Lehrpersonen auch das der Schüler*innen und setzen es in Bezug zur globalen Beziehungseinschätzung. In beiden Klassen ereigneten sich mehrheitlich neutrale bis positive Interaktionen. In der Klasse mit der aus Schüler*innensicht positiven LS-Beziehung verhielt sich die Lehrperson häufiger freundlich, in der Klasse mit der negativen LS-Beziehung konnten hingegen auch unfreundliche oder gar feindselige Interaktionen zwischen der Lehrperson und den Schüler*innen beobachtet werden.

An diesen Forschungsarbeiten und Erkenntnissen setzt die vorliegende Studie an. Untersucht wird der Zusammenhang zwischen den beobachteten unterrichtlichen Interaktionen und den Beziehungseinschätzungen sowohl der Schüler*innen wie auch der Lehrpersonen, da diese subjektiv sind und z. T. nicht übereinstimmen. Es kommt eine im deutschsprachigen Raum bisher kaum eingesetzte Methode, die State Space Grids Methode, zur Analyse sozialer Interaktionen zum Einsatz. Die gewonnenen Erkenntnisse können zum Aufbau und zur Förderung positiver unterrichtlicher Interaktionen und LS-Beziehungen für die Aus- und Weiterbildung von Lehrpersonen von Bedeutung sein. So zum Beispiel für die Reflexion von positiv und negativ eingeschätzten LS-Beziehungen und wie diese mit dem Verhalten bzw. den unterrichtlichen Interaktionen zusammenhängen.

\section{Theoretischer Hintergrund}

\subsection{Soziale Interaktionen als Grundbausteine der Beziehung}

Lehrpersonen und Schüler*innen sind sich wechselseitig beeinflussende Partner*innen in einem sozialen System (Herzog 2009). Die Theorie Dynamischer Systeme (Granic und Hollenstein 2003; Koopmans und Stamovlasis 2016) geht davon aus, dass sich die Einheiten eines Systems gegenseitig beeinflussen und da- 
durch Veränderungen im System hervorrufen (van Geert 2009). Entwicklungen und Veränderungen eines Systems sind somit nicht auf die isolierten Verhaltensweisen ihrer Teilsysteme zu reduzieren, sondern das Verhalten wird in Relation zueinander betrachtet (Koopmans und Stamovlasis 2016). Demnach steht nicht das Verhalten einzelner Akteure im Zentrum, sondern deren wechselseitiges Verhalten bzw. die soziale Interaktion.

Auf Grundlage sozialer Interaktionen entstehen und entwickeln sich Beziehungen (Hinde 1997; Granic und Hollenstein 2003; Hollenstein 2007; Wubbels et al. 2015, 2016). Beziehungen sind fortdauernde Verbindungen zwischen Individuen, die sich u. a. durch unterschiedliche zeitliche Dauer (Kontinuität) und eine gemeinsame Geschichte auszeichnen (Wentzel 2012). Tägliche Erfahrungen auf der Mikroebene lassen eine Beziehungseinschätzung auf der Makroebene entstehen oder verändern diese (Pianta et al. 2003). Somit bilden und verändern unterrichtliche Interaktionen eine mehr oder weniger beständige LS-Beziehung (Wubbels et al. 2015) und diese beeinflusst das interpersonale Verhalten (Granic und Hollenstein 2003; Mainhard et al. 2012; Pennings et al. 2014b). Das heißt, wie Lehrperson und Schüler*innen im Unterricht interagieren, beeinflusst die Beziehungseinschätzung und diese beeinflusst wiederum die zukünftigen Interaktionen im Unterricht.

\subsection{Unidirektionale Betrachtung pädagogischer Interaktionen}

Pädagogische Interaktionen können unidimensional oder interaktional bzw. transaktional betrachtet und erforscht werden (Thies 2017). Während unidirektionale Forschungszugänge primär vom Verhalten der Lehrperson oder der Schüler*innen ausgehen, stellen interaktionistische und transaktionale Zugänge die Interaktion ins Zentrum.

Tausch und Tausch (1973) untersuchten das Verhalten von Lehrpersonen in pädagogischen Interaktionen. Sie entwickelten in Anlehnung an die Erziehungsstilforschung ein zweidimensionales Konzept mit einer Beziehungs- (emotionale Wärme vs. Kälte) und einer Lenkungsdimension (starke vs. geringe Lenkung). Ein ähnliches zweidimensionales Modell stammt von Wubbels et al. (2005, 2006), wonach sich jedes Verhalten von Lehrpersonen durch eine Lenkungsdimension (Influence: dominance vs. submission) und eine Nähedimension (proximity: opposition vs. cooperation) beschreiben lässt. In neueren Arbeiten wurden die Begriffe Influence und Proxmity durch Agency und Communion ersetzt (Gurtman 2009; Wubbels et al. 2015). Agency meint Streben nach Einfluss und Kontrolle und Communion beschreibt die menschliche Wärme. ,Agency suggests that someone is becoming individuated, dominant, has power and control, whereas communion means someone is social, shows love union, friendliness, and affiliation“ (Wubbels et al. 2015, S. 366).

Die Forschungsgruppe um Wubbels (Wubbels und Brekelmans 2005; Wubbels et al. 2006) untersuchte den Zusammenhang zwischen dem Verhalten von Lehrpersonen und den Schulleistungen und der Motivation von Schüler*innen. Sie konnten zeigen, dass Schüler*innen, welche bei der Lehrperson eine größere Kontrolle wie auch mehr Wärme wahrnahmen, bessere kognitive Leistungen, ein stärkeres Engagement und positivere fachbezogene Einstellungen zeigten. Das Verhalten erfolgreicher Lehrpersonen zeichnet sich demnach durch die Kombination einer hohen 
Agency mit einer hohen Communion aus (Wubbels et al. 2015). Eine hohe Agency meint hier nicht kontrollierendes oder striktes Lehrpersonenverhalten, sondern die Gestaltung einer strukturierten sozialen Umgebung mit klaren Erwartungen, welche die Autonomie der Schüler*innen unterstützt. Eine hohe Communion vermittelt den Schüler*innen Anerkennung und Respekt, was wiederum für die Akzeptanz von Regeln förderlich ist. Eine solche Lernumgebung unterstützt die Autonomieentwicklung und das Engagement der Schüler*innen (Wubbels et al. 2015). Obwohl sich die Unterrichtsqualitätsforschung nicht als ,direkte Fortführung der Erziehungsstilforschung“ (Einsiedler 2017, S. 278) versteht, zeigen sich doch konzeptionelle Bezüge zu den Dimensionen soziale Unterstützung und Klassenführung, bei denen es auch um Gestaltung einer strukturierten Lernumgebung und einen respektvollen und wertschätzenden Umgang im Unterricht geht.

\subsection{Erforschung pädagogischer Interaktionen}

Eine Methode, um Interaktionen nicht unidirektional, sondern interaktional, in ihrer Wechselseitigkeit und über die Zeit zu erforschen, stellen State Space Grids (SSG) dar (Hollenstein 2013; Lamey et al. 2004). Es handelt sich hier um eine Methode, die es ermöglicht das wechselseitige Verhalten von Interaktionspartner*innen bzw. die aufeinanderfolgenden Interaktionen im zeitlichen Verlauf grafisch darzustellen und zu analysieren. Die SSG-Methode wurde bisher zur Erforschung von ElternKind-Interaktionen (Granic und Lamey 2002; Granic et al. 2003, 2007; Hollenstein et al. 2004) und zur Erforschung von Interaktionen unter Vorschulkindern (Martin et al. 2005) eingesetzt. In neueren Forschungsarbeiten werden zunehmend auch die im Unterricht ablaufenden pädagogischen Interaktionen in den Fokus genommen (Mainhard et al. 2012; Pennings et al. 2014b; Pennings und Hollenstein 2019; Pennings und Mainhard 2016). Dazu wurde das interpersonale Verhalten im Unterricht aus einer Beobachtendenperspektive auf einer mikrogenetischen Ebene mittels hochinferenter Kategorien für Communion und Agency mit der SSG-Methode analysiert und mit makrogenetischen Fragebogendaten in Beziehung gesetzt. Mainhard et al. (2012) analysierten zusätzlich zum Verhalten der Lehrperson auch das der Schüler*innen. Sie untersuchten zwei Klassen, eine in der die Schüler*innen die Beziehung zur Lehrperson positiv (A) und eine, in der sie diese negativ (B) einschätzten. Die Ergebnisse zeigen, dass sich die Mehrheit der pädagogischen Interaktionen in den untersuchten Klassen in einem neutralen bis positiven Bereich abspielten. Zudem verhielt sich in Klasse A die Lehrperson häufiger freundlich und nur in Klasse B konnten gegenseitig unfreundliches oder gar feindseliges Verhalten beobachtet werden. Auch in anderen Studien spielte sich, unabhängig von der LSBeziehungseinschätzung der Schüler*innen, ein Großteil der LS-Interaktionen in einem neutralen bis positiven Bereich ab (Pennings und Mainhard 2016; Pennings et al. 2014b).

Mainhard et al. (2012) gingen weiter der Frage nach, welche Bedeutung der Variabilität in pädagogischen Interaktionen zukommt. In Studien zu Eltern-KindInteraktionen wird eine große Variabilität als positiv für die Eltern-Kind-Beziehung angesehen (Granic und Hollenstein 2003). Diese zeigt sich darin, dass die Interaktionen bzw. die Zustände im State Space häufig wechseln, was als Flexibilität oder 
Anpassung des interpersonalen Verhaltens gedeutet und als Indikator für die Qualität der Interaktionen angesehen wird (Granic et al. 2007; Hollenstein et al. 2004). Die Eltern passen demnach ihr Verhalten situativ an und verhalten sich nicht rigide. Die Ergebnisse von Mainhard et al. (2012) zu pädagogischen Interaktionen im Unterricht gehen allerdings in die andere Richtung. In jenen Klassen mit einem weniger guten sozialen Klima zeigte sich eine größere Variabilität in den Interaktionen, d.h. es gab mehr Wechsel zwischen den Zellen im SSG. Dass die Beziehungseinschätzung negativ mit der Variabilität des Verhaltens der Lehrperson bzw. mit den pädagogischen Interaktionen zusammenhängt, zeigen auch andere Studien. So schätzten Schüler*innen die Communion von Lehrpersonen, die mehr Variabilität in ihrem interpersonalen Verhalten zeigten, niedriger ein (Pennings et al. 2014a; Pennings und Mainhard 2016). Auch auf interaktionaler Ebene hängt die Beziehungseinschätzung der Schüler*innen negativ mit der Variabilität der Interaktionen für Communion (Mainhard et al. 2012; Pennings et al. 2014b, 2018) und für Agency zusammen (Mainhard et al. 2012).

Der Forschungsüberblick hat gezeigt, dass es bisher wenig Forschung zu pädagogischen Interaktionen im Unterricht gibt und auch wenig Studien, welche die Methode State Space Grid zur Erforschung unterrichtlicher Interaktionen angewendet haben. Die meisten Studien hierzu stammen aus den Niederlanden und untersuchten entweder das Verhalten der Lehrpersonen in Kombination mit der globalen Beziehungseinschätzung der Schüler*innen oder recht kleine Stichproben (zwei Klassen). Wie die beobachteten pädagogischen Interaktionen mit den Beziehungseinschätzungen der Lehrpersonen zusammenhängen wurde bislang kaum erforscht. Zudem wurde die Methode State Space Grid unseres Wissens im deutschsprachigen Raum noch nicht zur Erforschung unterrichtlicher Interaktionen eingesetzt. Die vorliegende Studie setzt an diesen Erkenntnissen bzw. dieser Forschungslücke an und untersucht vor dem Hintergrund der Interpersonalen Theorie pädagogische Interaktionen und deren Variabilität im regulären Unterricht (Mikroebene) von Primarschulklassen der Stufe 5 und 6 für die Dimensionen Agency und Communion und den Zusammenhang mit globalen LS-Beziehungseinschätzungen (Makroebene) sowohl aus Schüler*innen- wie auch aus Lehrpersonensicht. Bei der Auswahl der Unterrichtsstunden wurde darauf geachtet, dass die Fächer Deutsch, Mathematik oder Natur Mensch Gesellschaft unterrichtet wurden.

\section{Fragestellungen}

Fragestellung 1 Wie verteilen sich die pädagogischen Interaktionen für Agency und Communion im State Space Grid?

Forschungsbefunde deuten darauf hin, dass sich ein Großteil der pädagogischen Interaktionen im Unterricht in einem neutralen bis positiven Bereich abspielt, und zwar unabhängig davon, wie die Beziehung von den Schüler*innen eingeschätzt wird (Mainhard et al. 2012; Pennings et al. 2014b; Pennings und Mainhard 2016). Es wird deshalb angenommen, dass die Interaktionen zwischen Lehrperson und Schüler*innen während einer Unterrichtsstunde sachlich-neutral bis freundlich sind und nicht einseitig gelenkt oder kontrolliert werden. 
Fragestellung 2 Inwiefern korreliert das im Unterricht beobachtete Verhalten von Lehrpersonen und Schüler*innen bzw. deren Interaktionen für die Dimensionen Agency und Communion mit der LS-Beziehungseinschätzung aus Perspektive von Lehrpersonen und Schüler*innen?

Es wird angenommen, dass in Klassen, in denen die LS-Beziehung schlechter eingeschätzt wurde, auch mehr ungünstige Interaktionen im Unterricht beobachtet werden können. Z.B. indem weniger wertschätzend und freundlich miteinander umgegangen wird und/oder die Lehrperson das Verhalten der Lernenden stark kontrolliert und lenkt.

Fragestellung 3 Wie hängt die Variabilität der pädagogischen Interaktionen im Unterricht mit der Einschätzung der LS-Beziehung zusammen?

Es wird die Hypothese überprüft, dass ein negativer Zusammenhang zwischen der Variabilität des interpersonalen Verhaltens und der Beziehungseinschätzung der Schüler*innen besteht.

\section{Methode}

Die Fragebogendaten und die Videoaufnahmen, welche im Rahmen der vorliegenden Studie analysiert wurden, stammen aus dem Forschungsprojekt „Wahrnehmung sozialer Interaktionen im Unterricht“". Im Rahmen dieses Projekts wurden an 83 Primarschulklassen der Mittelstufe (5. und 6. Klasse) in der Schweiz globale Daten zu Unterrichtsstörungen, der LS-Beziehung und Klassenführung mit Fragebogen erfasst (für Details vgl. Wettstein, Ramseier et al. 2016, 2018; Wettstein, Scherzinger et al. 2016, 2018). An der Fragebogenerhebung nahmen 1290 Schüler*innen (48,2\% Mädchen, $M=11,47$ Jahre alt, $S D=0,77)$ und 83 Klassenlehrpersonen $(65,1 \%$ weiblich, $M=39,5$ Jahre alt, $S D=11,78$ ) teil. Für die vorliegende Studie sind die globalen Daten zur pädagogischen Beziehung aus Sicht der Lehrpersonen wie auch der Schüler*innen (Makroebene) zentral. Lehrpersonen- und Schüler*innenfragebogen enthalten inhaltsgleiche Items. In beiden Versionen umfasste die Skala „LS-Beziehung“ sechs Items (z. B. „Ich mag diese Lehrperson“, „Ich verstehe mich gut mit dieser Lehrperson“, „Diese Lehrperson unterstützt uns bei Problemen“, „Sie ist eine gute Lehrperson"; vierstufige Antwortskala; Cronbachs $\alpha=0,88$; für Details vgl. Wettstein, Ramseier et al. 2016, 2018). Die Urteile der Schüler*innen wurden für jede Klasse aggregiert. Die Intraklassenkorrelation für die Skala Beziehung wies ein ICC der latenten Variablen von 0,33 auf (Wettstein, Ramseier und Scherzinger 2018).

Im Anschluss an die Fragebogenerhebung wurden an 18 Klassen jeweils drei Unterrichtsstunden gefilmt und hinsichtlich Unterrichtsstörungen ausgewertet (für weiterführende Informationen siehe Scherzinger et al. 2018). Bei der Auswahl der Unterrichtsstunden wurde darauf geachtet, dass Deutsch, Mathematik oder Natur, Mensch, Gesellschaft unterrichtet wurden und keine musisch-gestalterischen Fächer oder Sport. An der Videostudie nahmen 272 Schüler*innen, 18 Klassen- sowie 18 Fachlehrperson teil. Die Lehrpersonen, die Schüler*innen sowie ihre gesetzlichen Vertreter*innen willigten in die Videoerhebung ein. 


\subsection{Stichprobe}

Für die vorliegende Studie wurden aus dem Projekt „Wahrnehmung sozialer Interaktionen im Unterricht", d.h. aus den 18 Klassen der Videostudie, 8 Klassen bzw. 16 Lehrpersonen (zwei Lehrpersonen pro Klasse) ausgewählt. Die Auswahl der Klassen erfolgte im Sinne eines qualitativen Stichprobenplans kriteriengeleitet (Kelle und Kluge 2010), und zwar über die globalen Beziehungseinschätzungen aus der Fragebogenstudie. Da Schüler*innen und Lehrpersonen die Beziehung z. T. sehr unterschiedlich eingeschätzt haben, wurden bei der Fallauswahl sowohl die Schüler*innen- wie auch die Lehrpersonenperspektive berücksichtigt, was vier mögliche Kombinationen ergab.

- konvergent positiv bzw. negativ (Lehrperson und Schüler*innen schätzten die LSBeziehung ähnlich positiv oder negativ ein)

- divergent 1 und divergent 2 (Entweder schätzt die Lehrperson die LS-Beziehung positiv und die Schüler*innen negativ ein (divergent 1) oder umgekehrt (divergent 2)).

Alle 18 Klassen der Videostudie wurden einer dieser vier Gruppen zugeteilt. Für die vorliegende Studie wurden anschließend aus jeder Gruppe jeweils zwei Klassen mit den extremsten Werten ausgewählt.

Insgesamt wurde je eine Unterrichtsstunde (à $45 \mathrm{~min}$ ) von 16 Lehrpersonen an acht Primarschulklassen aus dem Kanton Bern ( $n=126$ Schüler*innen; $M=11,5$ Jahre; 42,1\% weiblich) untersucht. Die Stichprobe bestand aus drei 5. Klassen, vier 6. Klassen und einer Mehrjahrgangsklasse (5. und 6. Klasse). Bei den 16 Lehrpersonen handelt es sich um $n=8$ Klassenlehrpersonen (37,5\% weiblich, $M=41,13$ Jahre alt, $S D=12,4)$ und $n=8$ Fachlehrperson $(75,0 \%$ weiblich, $M=40,6$ Jahre alt, $S D=8,1)$. Von jeder der acht Klassen waren somit die Klassen- und eine Fachlehrperson beteiligt. Die Klassenlehrpersonen unterrichteten im Durchschnitt $M=21,9$ $(S D=3,5)$ Unterrichtsstunden pro Woche an der untersuchten Klasse und insgesamt $M=23,9(S D=3,6)$ Unterrichtsstunden pro Woche an der Schule. Sechs der acht Klassenlehrpersonen (75,0\%) wiesen mehr als sechs Jahre Berufserfahrung auf. Die Fachlehrpersonen unterrichteten im Durchschnitt $M=4,9(S D=1,6)$ Unterrichtsstunden pro Woche an der untersuchten Klasse und $M=14,6(S D=4,9)$ Unterrichtsstunden an der Schule. Alle acht Fachlehrpersonen wiesen mehr als sechs Jahre Berufserfahrung auf.

\subsection{Durchführung}

Der Unterricht wurde mit zwei GoPro-Videokameras und einem Diktafon aufgezeichnet, um mögliche Reaktivitätseffekte durch die Anwesenheit von Forschenden auf Seiten der Schüler*innen sowie der Lehrpersonen zu minimieren. Jeweils eine Kamera wurde an der vorderen und an der hinteren Breite des Klassenzimmers befestigt. Nach einer Eingewöhnungszeit wurden je drei aufeinanderfolgende Unterrichtsstunden meist ab der ersten Stunde am Morgen gefilmt. Hinsichtlich Unterrichtsplanung gab es keine Vorgaben. Der Unterricht sollte von der Lehrperson so wie üblich durchgeführt werden. Bei der Auswahl des Filmtags wurde darauf geach- 
tet, dass an diesem Morgen Deutsch, Mathematik oder Natur Mensch Gesellschaft und keine musisch-gestalterischen Fächer oder Sport unterrichtet wurden.

\subsection{Kategoriensystem und Kodierung}

Für die Kodierung der Videos wurde mittels deduktiver und induktiver Vorgehensweise ein Kategoriensystem entwickelt. Theoretische Grundlage für die Erstellung der fünfstufigen Kategorien bildeten frühere Studien, in denen das interpersonale Verhalten mittels Agency und Communion erhoben wurde (Mainhard et al. 2012; Pennings et al. 2014b; Wubbels et al. 2016). Das entwickelte Kategoriensystem wurde am Material induktiv angepasst, ergänzt und ausdifferenziert. Der Vorteil der Dimensionen Agency und Communion liegt darin, dass sie einer kommunikationspsychologischen Tradition folgend, das interpersonale Verhalten von Interaktionspartner*innen beschreiben. Sie können somit sowohl für die Kodierung des Verhaltens der Lehrperson wie der Schüler*innen angewandt werden.

Die Dimension Agency umfasst fünf Kategorien: (1) „sehr passiv“ z. B. reagiert oder antwortet nicht, greift nicht ein bzw. versucht nicht zu lenken oder die Schüler*innen verhalten sich sehr passiv, (2) „passiv“, (3) „,reagierend“, (4) ,aktiv/den Unterricht gestaltend“ z.B. die Schüler*innen sind aktiv oder zeigen Eigeninitiative und (5) ,sehr aktiv bzw. dominant“ z.B. bestimmend, kontrollierend, befiehlt, unterbricht Schüler*innen (für Details siehe Tab. 4 im Anhang). Die Kategorien für Communion lauten: (1) „feindlich“ z. B. beleidigt, stellt Schüler*innen bloß oder Schüler*innen verhalten sich feindselig gegenüber der Lehrperson, (2) ,unfreundlich“, (3) „,sachlich-nüchtern“, (4) „,freundlich“ und (5) ,sehr freundlich bzw. kumpelhaft" z. B. übertrieben freundlich, witzelt, professionelle Distanz wird nicht gewahrt oder die Schüler*innen verhalten sich sehr kumpelhaft, distanzlos gegenüber der Lehrperson. Die höchste Ausprägung (5) bei Communion bedeutet nicht etwa „,besser“ oder wünschenswerter, wie das Beispiel „,kumpelhafter Umgang“ zeigt. Als lernförderlich wird ein neutraler bis freundlicher Umgang angesehen. Auch eine sehr hohe Agency (5; z. B. kontrollierend/strikt) wird nicht als förderlich betrachtet. Eine hohe Agency hingegen, die nicht kontrollierend oder strikt ist und die Autonomieentwicklung der Schüler*innen unterstützt wird in Kombination mit einer hohen Communion als förderlich für das Lernen der Schüler*innen angesehen (Wubbels et al. 2015).

Die Kodierung des Interaktionsverhaltens von Lehrpersonen und Schüler*innen im Unterricht erfolgte mithilfe des entwickelten Kategoriensystems. Dazu wurde jede videografierte Unterrichtsstunde viermal nach vorab festgelegten Kodierregeln in MAXQDA 2018 kodiert, und zwar für jede Dimension (Agency und Communion) sowohl für die Schüler*innen als auch für die Lehrperson einzeln. Als Kodierregeln wurde beispielsweise festgehalten, dass das Verhalten der Lehrperson und der Schüler*innen unabhängig voneinander kodiert wird und wenn einzelne Schüler*innen direkt mit der Lehrperson interagieren, jeweils dieses Verhalten und nicht das der Klasse kodiert wird.

Das Verhalten der Lehrperson oder der Schüler*innen wurde kontinuierlich, d.h. sobald und solange es auftritt, auf der fünfstufigen Skala für Communion und in einem zweiten Durchgang für Agency kodiert. Wenn das Verhalten änderte, wurde 
dieses jeweils einem anderen Kode zugeordnet. Das Vorgehen für das Verhalten der Schüler*innen war analog. Insgesamt wurden 5570 Kodes vergeben, wobei 2722 Kodierungen auf die Schüler*innen und 2848 auf die Lehrpersonen fielen.

Zur Bestimmung der Interraterreliabilität wurden 9\% des Datenmaterials durch einen unabhängigen Rater zweitkodiert und anschließend Sekunde für Sekunde mit den Kodierungen des ersten Raters verglichen. Die prozentuale Übereinstimmung lag durchschnittlich bei $87,25 \%$. Als Maß für die zufallskorrigierte Übereinstimmung wurde Cohen's Kappa $(\kappa=0,65)$ berechnet.

\subsection{Datenanalyse}

Die kodierten Daten wurden aus MAXQDA exportiert und in Excel, zur Erstellung der State Space Grids, aufbereitet. Mit dem Programm GridWare 1.15 (Lamey et al. 2004) wurden für jede Klasse SSGs erstellt, in denen die Interaktionen zwischen zwei Individuen oder zwei Gruppen in einem zweidimensionalen System grafisch dargestellt werden. In der vorliegenden Studie bildete die Agency bzw. die Communion der Lehrperson die x-Achse, die der Schüler*innen die y-Achse (vgl. Abb. 1), woraus sich für jede Klasse ein SSG für Agency und eines für Communion ergaben. Ein Kategoriensystem mit fünf Kategorien auf jeder Achse ergibt ein SSG mit 25 Zellen, welches alle möglichen Kombinationen von Verhaltensweisen beider Parteien abbildet.

Zeitlich synchrone Verhaltensweisen werden auf dem zweidimensionalen Raster, bei dem die möglichen Verhaltensweisen der beiden Parteien zwei orthogonale Achsen bilden, mit einem Punkt abgebildet. Die Größe des Punkts stellt die Dauer der Interaktion in der jeweiligen Zelle dar (je größer der Punkt, desto länger dauert die Interaktion). Verändert sich das Verhalten einer oder beider Parteien, entsteht ein neuer Punkt auf dem Raster, der durch einen Pfeil mit dem vorherigen verbun-
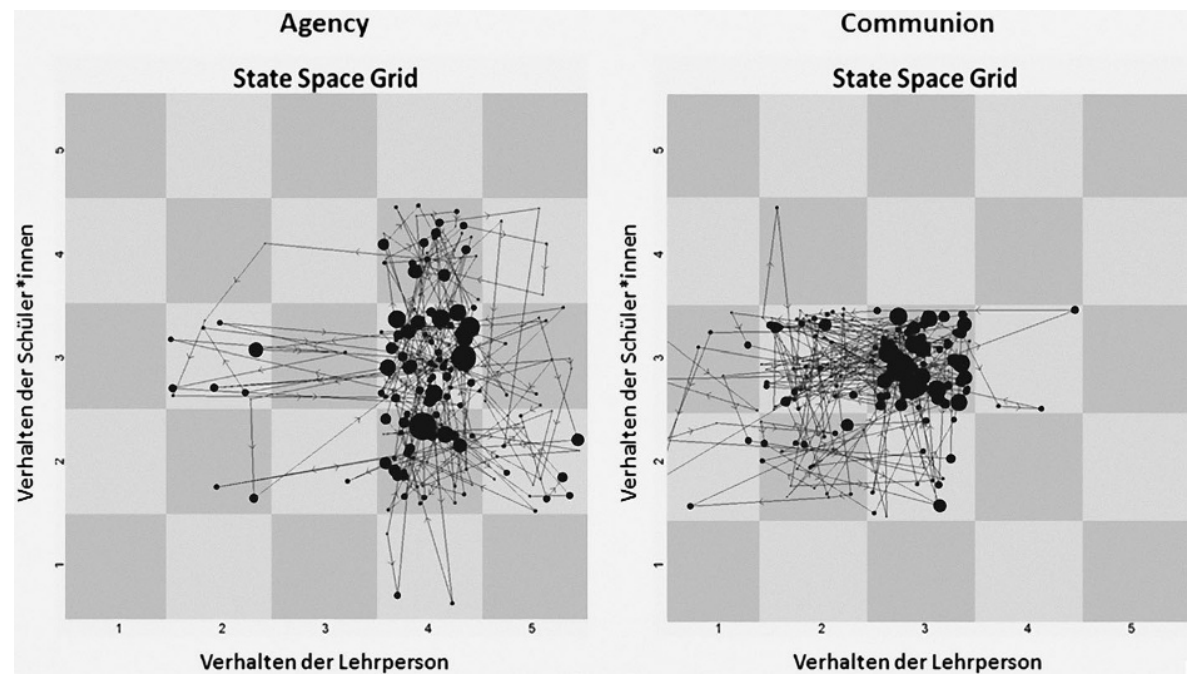

Abb. 1 State Space Grid für eine Klasse während einer Unterrichtsstunde für Agency und Communion 
den ist. Die Analyse zeigt, in welchen Zellen sich die pädagogischen Interaktionen abspielen und wie lange diese dauern.

Für die Korrelationsanalysen wurden zuerst die prozentualen zeitlichen Anteile der Interaktionen für jede Zelle berechnet. Dazu wurde die zeitliche Dauer der Interaktionen für jede der 25 Zellen im SSG durch die Dauer einer Unterrichtsstunde (45 min) geteilt. Für die Überprüfung des Zusammenhangs zwischen den zeitlichen Interaktionsanteilen für jede Zelle im SSG und den globalen Beziehungseinschätzungen der Schüler*innen sowie der Lehrpersonen aus der Fragebogenerhebung wurden Produkt-Moment-Korrelationen berechnet. Die Auswertung erfolgte für beide Dimensionen, Agency und Communion, und auch für die Beziehungseinschätzung der Lehrpersonen und der Schüler*innen getrennt.

Die strukturelle Analyse liefert Informationen zur Struktur der Interaktionen (Variabilität) und zeigt, wie variabel bzw. stabil sich die pädagogischen Interaktionen im Unterricht gestalten. Variabilität bedeutet, dass die Interaktionen im SSG den Zustand wechseln und dementsprechend Zellwechsel zu beobachten sind. Zu einem Zellwechsel kommt es, wenn sich das Verhalten von mindestens einem Akteur ändert (z.B. von sachlich-neutralem zu unfreundlichem Verhalten). Für die Variabilität der Interaktionen sind folgende Maße wichtig: Anzahl Wechsel zwischen den Zellen, Anzahl besuchter Zellen (Zellumfang) und Dispersion (Hollenstein 2013). Bei der Dispersion handelt es sich um einen relativen Wert, der den Zellumfang in Bezug zur zeitlichen Dauer in der Zelle setzt. Die zeitliche Dauer wie auch die Maße der Variabilität (Anzahl Wechsel zwischen den Zellen, absolute Anzahl besuchter Zellen und Dispersion; Granic et al. 2007) werden in der Software Gridware angezeigt.

\section{Ergebnisse}

\subsection{Forschungsfrage 1: Verteilung der Interaktionen im State Space Grid}

Die Ergebnisse für Agency zeigen (vgl. Abb. 2), dass über alle Klassen hinweg in rund $70 \%$ der Interaktionen die Schüler*innen und die Lehrpersonen über eine mittlere (3) bis hohe (4) Agency verfügten. In 32\% der Interaktionen hatten die Lehrpersonen eine etwas größere Agency als die Schüler*innen (Zelle 43; x-Achse Lehrperson (4), y-Achse Schüler*innen (3)), in $17 \%$ die Schüler*innen (Zelle 34). In $21 \%$ der Interaktionen wiesen die Lehrpersonen eine relativ niedrige Agency (1 oder 2) auf, sie kontrollierten oder lenkten kaum, die Schüler*innen hingegen zeigten eine mittlere bis sehr hohe Agency (Zellen 15, 23, 24, 25). Im Vergleich dazu hatten die Lehrpersonen in nur rund $2 \%$ der Interaktionen eine deutlich höhere Agency als die Schüler*innen (Zellen 41, 42, 51, 52).

Für Communion zeigte sich, dass in $75 \%$ der Interaktionen sowohl die Lehrperson als auch die Schüler*innen sachlich-nüchtern bis freundlich miteinander umgingen, d.h. eine mittlere bis hohe Communion aufwiesen (Zellen 33, 34, 43, 44) (vgl. Abb. 2). Dass sich eine der Parteien unfreundlich verhielt (C2), kam in insgesamt rund $23 \%$ der Interaktionen vor, davon verhielten sich in $19 \%$ der Interaktionen die Schüler*innen unfreundlich gegenüber der Lehrperson (Zelle 32). In ungefähr $4 \%$ der Interaktionen waren allerdings auch die Lehrpersonen unfreundlich (Zel- 

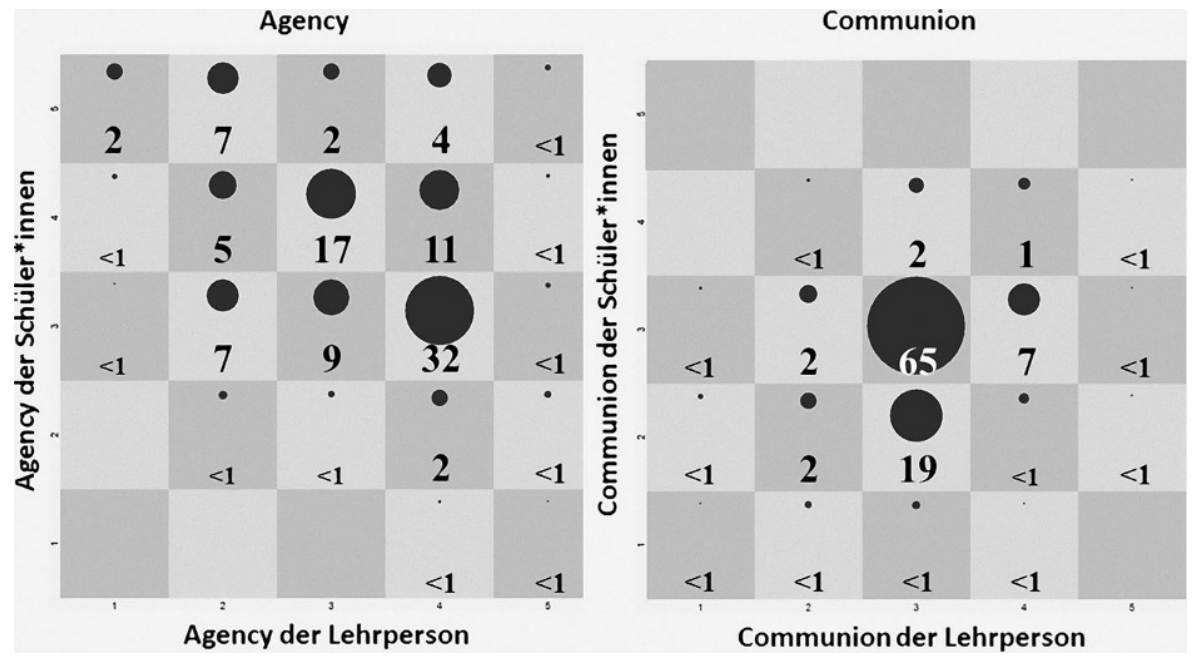

Abb. 2 Prozentuale Verteilung der Interaktionen aller Klassen für Agency und Communion. (Die Größe der Punkte und die Zahl zeigen den prozentualen zeitlichen Anteil an Interaktionen in der jeweiligen Zelle)

len 22, 23). Sehr selten bzw. nur in Einzelfällen wurde feindseliges Verhalten (C1) der Lehrperson bzw. der Schüler*innen oder kumpelhaftes, distanzloses Verhalten (C5) der Lehrperson beobachtet.

Hypothese 1, dass die Interaktionen zwischen zwischen Lehrperson und Schüler*innen während einer Unterrichtsstunde überwiegend in Bereichen einer mittleren bis hohen Communion bzw. Agency stattfinden, konnte somit bestätigt werden.

\subsection{Forschungsfrage 2: Beziehungseinschätzung und pädagogische Interaktionen}

Die Korrelationsanalysen haben ergeben (vgl. Tab. 1), dass eine positive globale Beziehungseinschätzung aus Schüler*innensicht positiv mit beobachteten mikrogenetischen Interaktionen korreliert, bei denen die Lehrperson eine mittlere und die Schüler*innen eine etwas höhere Agency besaßen (Zelle 34; $r=0,50, p<0,05$ ). Ein negativer Zusammenhang zeigte sich hingegen zwischen der Beziehungseinschätzung der Schüler*innen und Interaktionen, bei denen die Lehrperson eine hohe, die Schüler*innen eine deutlich niedrige Agency aufwiesen (Zelle 42; $r=-0,50, p<0,05$ ). Demnach hängt eine negative Beziehungseinschätzung aus Schüler*innensicht mit beobachteten Interaktionen zusammen, die stark durch die Lehrpersonen kontrolliert und gelenkt werden, während die Schüler*innen selber eher passiv sind. Bei den Lehrpersonen korrelierte die globale Beziehungseinschätzung nicht statistisch signifikant mit den pädagogischen Interaktionen für Agency.

Für Communion (vgl. Tab. 2) hat sich für die Schüler*innen und die Lehrpersonen ein negativer Zusammenhang zwischen der Beziehungseinschätzung und Interaktionen ergeben, in denen sich mindestens eine Partei unfreundlich verhielt, positive 
Tab. 1 Korrelationen der prozentualen zeitlichen Anteile an Interaktionen für Agency und der Beziehungseinschätzung der Lehrpersonen bzw. der Schüler*innen

\begin{tabular}{lllllll}
\hline Agency & 5 & $-0,12 \mid-0,32$ & $-0,37 \mid-0,15$ & $-0,37 \mid 0,05$ & $-0,48 \mid-0,32$ & - \\
Schü- & 4 & - & $-0,02 \mid-0,18$ & $0,35 \mid \mathbf{0 , 5 0}$ & $-0,14 \mid-0,01$ & - \\
ler*innen & 3 & - & $-0,05 \mid-0,05$ & $0,11 \mid 0,37$ & $0,17 \mid-0,13$ & - \\
& 2 & - & - & - & $-0,21 \mid-\mathbf{0 , 5 0 *}$ & - \\
& 1 & - & - & - & - & - \\
& & 2 & 3 & 4 & 5 \\
& & & & & \\
\hline
\end{tabular}

r Lehrperson I r Schüler*innen; Zellen mit weniger als $1 \%$ der Interaktionen sind durch - gekennzeichnet (1) „sehr passiv“, (2) „passiv“, (3) „,reagierend“, (4) ,,aktiv/den Unterricht gestaltend“, (5) „,sehr aktiv/ dominant"

$* p<0,05$

Tab. 2 Korrelationen der prozentualen zeitlichen Anteile an Interaktionen für Communion und der Beziehungseinschätzung der Lehrpersonen bzw. der Schüler*innen

\begin{tabular}{|c|c|c|c|c|c|c|}
\hline \multirow{7}{*}{$\begin{array}{l}\text { Communion } \\
\text { Schü- } \\
\text { ler*innen }\end{array}$} & 5 & - & - & - & - & - \\
\hline & 4 & - & - & $0,10 \mid \mathbf{0 , 6 0 *}$ & $0,32 \mid 0,49$ & - \\
\hline & 3 & - & $-0,38 \mid-\mathbf{0 , 5 6 *}$ & $0,37 \mid 0,18$ & $0,18 \mid \mathbf{0 , 5 4} *$ & - \\
\hline & 2 & - & $-0,54 * \mid-0,80 * *$ & $-0,30 \mid-0,23$ & - & - \\
\hline & 1 & - & - & - & - & - \\
\hline & & 1 & 2 & 3 & 4 & 5 \\
\hline & & & ion Lehrperson & & & \\
\hline
\end{tabular}

r Lehrperson I r Schüler*innen; Zellen mit weniger als $1 \%$ der Interaktionen sind durch - gekennzeichnet (1) „feindlich“, (2) „unfreundlich“, (3) „sachlich-nüchtern“, (4) „freundlich“ und (5) „sehr freundlich/ kumpelhaft"

$* p<0,05, * * p<0,01$

Korrelationen hingegen, wenn beide Parteien sachlich-nüchtern bis freundlich miteinander umgingen.

Die Ergebnisse zeigen, dass eine positive LS-Beziehung aus Sicht der Schüler*innen positiv mit Interaktionen korreliert, bei denen sowohl die Schüler*innen wie auch die Lehrperson eine mittlere bis hohe Communion zeigten (Zelle 34; $r=0,60, p<0,05 ;$ Zelle 43; $r=0,54, p<0,05)$. Ein negativer Zusammenhang ergab sich zwischen der Beziehungseinschätzung der Schüler*innen und Interaktionen, in denen sich entweder die Lehrperson (Zelle 23; $r=-0,56, p<0,05$ ) oder beide Parteien unfreundlich verhielten (Zelle 22; $r=-0,80, p<0,01$ ). Beidseitiges unfreundliches Verhalten hängte auch negativ mit der Beziehungseinschätzung der Lehrpersonen (Zelle 22; $r=-0,54, p<0,05)$ zusammen. Bei den Lehrpersonen zeigten sich keine weiteren statistisch signifikanten Ergebnisse.

Hypothese 2 konnte teilweise bestätigt werden. Für Communion zeigten sich, wie erwartet, negative Zusammenhänge zwischen der Beziehungseinschätzung und Interaktionen, die sich durch wenig wertschätzendes oder unfreundliches Verhalten auszeichneten. Für Agency zeigte sich ein negativer Zusammenhang zwischen der Beziehungseinschätzung der Schüler*innen und einer hohen Agency (4) der Lehrperson, jedoch nicht, wie angenommen, mit einer sehr hohen Agency (5), in Kombination mit einer niedrigeren Agency der Schüler*innen. 


\subsection{Forschungsfrage 3: Beziehungseinschätzung und Variabilität der pädagogischen Interaktionen}

Bei den Schüler*innen zeigte sich entgegen der Annahme keine statistisch signifikante Korrelation zwischen der Beziehungseinschätzung und der Variabilität ${ }^{1}$ des interpersonalen Verhaltens im Unterricht. Im Gegensatz dazu korrelierte die Beziehungseinschätzung der Lehrpersonen statistisch signifikant negativ mit der Anzahl Wechsel zwischen den Zellen im SSG (vgl. Tab. 3). Dieser negative Zusammenhang zeigte sich sowohl für Agency $(r=-0,60, p<0,05)$ wie auch für Communion $(r=-0,52, p<0,05)$. Zusätzlich korrelierte die Beziehungseinschätzung der Lehrpersonen negativ mit der Dispersion für die Agency $(r=-0,54, p<0,05)$. Hypothese 3 konnte somit nicht bestätigt werden. Für die Lehrpersonen zeigte sich allerdings ein negativer Zusammenhang zwischen der Variabilität interpersonalen Verhaltens und der Beziehungseinschätzung.

\section{Diskussion}

Im Zentrum der vorliegenden Studie steht der Zusammenhang zwischen der LSBeziehung, global erfasst mit Fragebogen, und den im Unterricht ablaufenden mikrogenetischen Interaktionen. Diese wurden videografiert und mit State Space Grids analysiert, welche in Kombination mit den Fragebogeneinschätzungen vertiefte Erkenntnisse zur Förderung und Entwicklung positiver unterrichtlicher Interaktionen und pädagogischer Beziehungen liefern, die sowohl für die Forschung als auch für die Aus- und Weiterbildung von Lehrpersonen genutzt werden können.

Die Ergebnisse zur Verteilung der pädagogischen Interaktionen während einer Unterrichtsstunde zeigen, dass sich mehr als zwei Drittel in einem neutralen bis positiven Bereich bewegten. Sowohl die Lehrpersonen wie auch die Schüler*innen wiesen eine mittlere bis hohe Agency bzw. eine neutrale bis hohe Communion auf, der Umgang miteinander war sachlich-neutral bis freundlich und sowohl Schüler*innen wie auch Lehrpersonen verhielten sich aktiv. Dieser Befund deckt sich

Tab. 3 Korrelationen zwischen den Massen für die Variabilität der Interaktionen und der Beziehungseinschätzung der Schüler*innen $(B E Z S u S)$ und der Beziehungseinschätzung der Lehrpersonen $(B E Z L P)$

\begin{tabular}{lllllll}
\hline & Agency & & \multicolumn{3}{c}{ Communion } \\
Skala & \# Wechsel & Zellumfang & Dispersion & \# Wechsel & Zellumfang & Dispersion \\
\hline BEZ & $-0,37$ & $-0,49$ & $-0,32$ & $-0,29$ & $-0,47$ & $-0,11$ \\
SuS & & & & & \\
BEZ LP & $\mathbf{- 0 , 6 0 *}$ & $-0,45$ & $\mathbf{- 0 , 5 4 *}$ & $\mathbf{- 0 , 5 2 *}$ & $-0,43$ & $-0,44$ \\
\hline
\end{tabular}

\# Wechsel Anzahl Wechsel zwischen den Zellen, Zellumfang Anzahl besuchter Zellen, Dispersion Zellumfang kontrolliert nach relativer Dauer

$* p<0,05$

\footnotetext{
1 Maße für die Variabilität der Interaktionen sind die Anzahl Wechsel zwischen den Zellen, die Anzahl besuchter Zellen (Zellumfang) und die Dispersion (relativer Wert, der den Zellumfang in Bezug zur zeitlichen Dauer in der Zelle setzt) (Hollenstein 2013).
} 
mit anderen Studien (Mainhard et al. 2012; Pennings et al. 2014b; Pennings und Mainhard 2016). Zudem ergaben die Korrelationsanalysen, dass eine positive Beziehungseinschätzung aus Sicht der Schüler*innen mit freundlichen, wertschätzenden und respektvollen Interaktionen im Unterricht einhergingen, eine negative Beziehungseinschätzung mit unfreundlichen, abweisenden und distanzierten Interaktionen. Auch bei den Lehrpersonen hängte die Beziehungseinschätzung negativ mit beobachtetem gegenseitig unfreundlichem Verhalten im Unterricht zusammen. Dieser Befund stimmt mit Studienergebnissen von Pennings et al. (2014b) überein, die gezeigt haben, dass in der Klasse, in der die Schüler*innen die LS-Beziehung schlechter einschätzten, unfreundlicher miteinander umgegangen wurde. In der Klasse, in der die LS-Beziehung positiv eingeschätzt wurde, verhielt sich die Lehrperson im Unterricht auch dann freundlich, wenn die Schüler*innen ihr gegenüber unfreundlich waren. Dies deutet darauf hin, dass sie in ihrer Rolle als Lehrperson professionell auf das Verhalten der Schüler*innen reagierte (Pennings et al. 2018). Ein unterstützendes Unterrichtsklima ist eine zentrale Qualitätsdimension von Unterricht und eine wichtige Grundlage für das Lernen, die Motivation und das Erleben positiver Emotionen von Schüler*innen (Klieme et al. 2006; Praetorius et al. 2018).

Für Agency zeigte sich ein positiver Zusammenhang zwischen der Beziehungseinschätzung und einer mittleren Agency der Lehrperson mit einer etwas höheren der Schüler*innen im Unterricht. Die Lehrpersonen gestalteten demnach den Unterricht und die Schüler*innen waren sehr aktiv. Ein negativer Zusammenhang mit der Beziehung zeigte sich hingegen bei einer hohen Agency der Lehrperson kombiniert mit einer niedrigen der Schüler*innen, wenn also die Lehrpersonen das Unterrichtsgeschehen dominierten und die Schüler*innen sich passiv verhielten. Eine hohe Agency der Lehrperson ist allerdings nicht in jedem Fall negativ, so beispielsweise bei Frontalunterricht. Möglicherweise ist die Differenz oder die Passung zwischen dem Verhalten der Akteure im Unterricht viel entscheidender. Wie stark kontrolliert, lenkt oder dominiert die Lehrperson das Unterrichtsgeschehen und die Schüler*innen sind passiv oder schafft sie es, die Schüler*innen zu aktivieren? Hier lässt sich ein Bezug zu zwei der drei Dimensionen der Unterrichtsqualität herstellen, welche die Tiefenstruktur von Unterricht beschreiben (Klieme et al. 2006; Kunter und Ewald 2016). Neben der Klassenführung, bei der es um die Steuerung des Unterrichts und eine möglichst effektive Nutzung der Zeit geht, sollen die Schüler*innen auch kognitiv aktiviert und damit angeregt werden, sich mit dem Stoff auseinanderzusetzen.

LS-Beziehungen, in welchen beide Parteien über eine ausreichend hohe, aber nicht zu hohe Agency verfügen, sind egalitärer als LS-Beziehungen, in welchen Lehrpersonen das Klassengeschehen einseitig lenken und kontrollieren. Zudem haben Schüler*innen gerade in der frühen Adoleszenz zunehmend Autonomieansprüche, auf welche Lehrpersonen entweder abwehrend bis eskalativ (Eccles et al. 1991) oder zugestehend reagieren und den Schüler*innen mehr Autonomie ermöglichen. Schüler*innen, die mehr Autonomieunterstützung durch die Lehrperson erfahren, berichten mehr Freude und Interesse am Unterricht (Park et al. 2012). Wenn Lehrpersonen die Autonomie der Schüler*innen einschränken, sind die Schüler*innen weniger motiviert und erleben auch häufiger negative Emotionen (Assor et al. 2005). Insbesondere für die Motivation der Schüler*innen wäre Autonomie bzw. Selbstbestimmung zentral (Deci und Ryan 1985). Fragebogenstudien haben gezeigt, dass 
Schüler*innen, welche bei ihrer Lehrperson Kontrolle und auch Wärme wahrnahmen, bessere kognitive Leistungen, ein grösseres Engagement und positivere fachbezogene Einstellungen aufwiesen (Wubbels und Brekelmans 2005; Wubbels et al. 2006).

Insgesamt zeigen die Ergebnisse der vorliegenden Studie, dass die LS-Beziehungseinschätzungen der Schüler*innen stärker mit den im Unterricht ablaufenden (positiven und negativen) Interaktionen assoziiert sind als die der Lehrpersonen. Dies kann verschiedene Gründe haben. Aus inhaltlicher Sicht kann vermutet werden, dass a) die Schüler*innen im Unterricht weniger unter Handlungsdruck stehen als die Lehrpersonen und sie deshalb mehr Zeit haben, die Interaktionen zu beobachten (Wettstein 2013), b) sie sich in ihrer Beziehungseinschätzung stärker an den unterrichtlichen Interaktionen orientieren, während die LS-Beziehungseinschätzung der Lehrpersonen unabhängiger von diesen konkreten Interaktionen ist und sie vor dem Hintergrund ihrer professionellen Rolle weitere Aspekte berücksichtigen, c) die Lehrpersonen sich in ihrem Verhalten weniger stark durch die LS-Beziehungsqualität beeinflussen lassen, d) der Befund könnte auch auf messmethodische Gründe zurückgeführt werden, da die Einschätzungen der Schüler*innen sich auf eine einzelne Lehrperson beziehen, während die Lehrperson ihre z.T. sehr differenziellen Beziehungen zu einzelnen Schüler*innen zu einem globalen Klassenurteil zusammenfasst oder e) durch die Aggregation der Urteile der Schüler*innen werden individuelle Synkrasien, d.h. individuelle Wahrnehmungsunterschiede, herausgemittelt, während sich das Urteil der Lehrpersonen auf eine einzige Quelle bezieht (Kenrick und Funder 1988).

Die Annahme, dass die Beziehungseinschätzung der Schüler*innen negativ mit der Variabilität der Interaktionen korreliert, konnte nicht bestätigt werden. Es zeigte sich jedoch ein Trend in die erwartete Richtung, welcher auch in anderen Studien gefunden wurde (Mainhard et al. 2012; Pennings et al. 2014a, 2018; Pennings und Hollenstein 2019; Pennings und Mainhard 2016). Die Beziehungseinschätzung der Lehrpersonen korrelierte hingegen statistisch signifikant negativ mit der Variabilität der pädagogischen Interaktionen. Eine größere Variabilität ist mit einem weniger wünschenswerten sozialen Klima (Pennings und Mainhard 2016) bzw. interpersonalem Verhalten der Lehrperson (Pennings und Hollenstein 2019) verbunden. Variabilität bedeutet hier nicht Flexibilität und Anpassung, sondern die Interaktionen werden als ,chaotisch“ bzw. weniger konsistent und für das Lernen wenig förderlich gedeutet. Die negative Assoziation zwischen interaktionaler Variabilität und der Beziehungseinschätzung der Lehrperson könnte also darauf zurückgeführt werden, dass die Variabilität ein Symptom eines wenig stabilen, störanfälligen Interaktionssystems darstellt.

\subsection{Stärken und Limitation}

Die Stärke der vorliegenden Studie besteht einerseits in der interaktionalen Betrachtung des Unterrichtsgeschehens, andererseits in ihrer ökologischen Validität. Zudem wurde mit State Space Grids eine im deutschsprachigen Raum bisher kaum eingesetzte Methode zur Analyse pädagogischer Interaktionen im Unterricht eingesetzt. Beziehungen werden meist global mit Fragebogen erfasst, dabei bleibt unklar, 
wie sich LS-Beziehungen im Unterricht entwickeln und verändern und mit den tatsächlichen unterrichtlichen Interaktionen zusammenhängen. In der vorliegenden Studie wurden die pädagogischen Interaktionen im Unterricht mit SSG analysiert. Anschließend wurde untersucht, inwiefern diese mikrogenetischen Daten mit der makrogenetischen LS-Beziehungseinschätzung der Schüler*innen und der Lehrpersonen zusammenhängen. Somit gehen die Ergebnisse über eine globale Einschätzung der LS-Beziehung hinaus und liefern Erkenntnisse über den Zusammenhang zwischen der subjektiv erlebten Qualität der LS-Beziehung aus Sicht von Lehrpersonen sowie Schüler*innen und den pädagogischen Interaktionen im Unterricht.

Neben den Vorteilen dieses qualitativen Zugangs besteht eine Limitation in der kleinen Stichprobengröße ( $n=16$ Lehrpersonen), weshalb die Daten kaum verallgemeinerbar sind. Zudem können anhand von Korrelationsanalysen nur Aussagen über Zusammenhänge zwischen der LS-Beziehung und den Interaktionen im Unterricht, jedoch nicht über Kausalzusammenhänge gemacht werden. Eine weitere Limitation der Studie ist, dass nur 9\% des Datenmaterials von einem unabhängigen Rater zweitkodiert wurde und somit die Übereinstimmung bzw. die Interraterreliabilität nur auf Basis dieser $9 \%$ berechnet wurde.

\subsection{Implikationen und Schlussfolgerungen}

Die Erfassung pädagogischer Interaktionen mit State Space Grids eröffnet ein vertieftes Verständnis für die Entstehung und Entwicklung der LS-Beziehung und gibt Hinweise zu positiven und auch kritischen Interaktionen im Unterricht, welche zur Reflexion und zur Verbesserung der LS-Beziehung in der Aus- und Weiterbildung von Lehrpersonen genutzt werden können. Insbesondere auch für die Auseinandersetzung damit, was ein lernförderliches und unterstützendes Klima sowie positive Interaktionen im Unterricht auszeichnen, was zu einem positiven Klima beiträgt und welche Rolle dabei Lehrpersonen zukommt, liefert die Studie entscheidende Erkenntnisse. Aufgabe von Lehrpersonen ist es, ein Lernumfeld zu schaffen, in dem freundlich, wertschätzend und respektvoll miteinander umgegangen wird, auch wenn sich die Schüler*innen unfreundlich, distanziert oder gar feindselig gegenüber der Lehrperson verhalten. Zudem ist es erforderlich, die Klasse zu führen und den Schüler*innen Partizipationsmöglichkeiten zu gewähren.

Für die Stärkung der LS-Beziehung wäre es zudem spannend, Interaktionen in kritischen Phasen genauer zu untersuchen und Lehrpersonen dafür zu sensibilisieren, sich auch bei unfreundlichem oder feindseligem Verhalten von Schüler*innen nicht reziprok zu verhalten oder die Situation eskalieren zu lassen, sondern ihre Agency situativ anzupassen und gleichzeitig eine hohe Communion aufrechtzuerhalten. Zudem sollten insbesondere auch Studierende wie berufseinsteigende Lehrpersonen angeregt werden, das Verhältnis von Communion und Agency zu reflektieren und ihnen sollte aufgezeigt werden, dass die beiden Dimensionen keinen Widerspruch darstellen (Wettstein und Scherzinger 2019; Wubbels und Brekelmans 2005; Wubbels et al. 2006).

Interessante Ansatzpunkte für zukünftige Studien zur LS-Beziehung wären, die spezifischen Verläufe von positiven und kritischen Interaktionen näher zu untersuchen. Auch die Effekte der LS-Beziehung bzw. positiver und negativer Interaktionen 
auf die Schülerinnen und Schüler, d.h. auf verschiedene Merkmale im Lernprozess, könnten detailliert untersucht werden. Weiter könnte auch die Wirkrichtung zwischen der Beziehungseinschätzung und den pädagogischen Interaktionen längsschnittlich untersucht werden, um die Zusammenhänge zwischen der Mikro- und der Makroebene besser zu verstehen.

Funding Open access funding provided by University of Teacher Education of Bern

Open Access Dieser Artikel wird unter der Creative Commons Namensnennung 4.0 International Lizenz veröffentlicht, welche die Nutzung, Vervielfältigung, Bearbeitung, Verbreitung und Wiedergabe in jeglichem Medium und Format erlaubt, sofern Sie den/die ursprünglichen Autor(en) und die Quelle ordnungsgemäß nennen, einen Link zur Creative Commons Lizenz beifügen und angeben, ob Änderungen vorgenommen wurden.

Die in diesem Artikel enthaltenen Bilder und sonstiges Drittmaterial unterliegen ebenfalls der genannten Creative Commons Lizenz, sofern sich aus der Abbildungslegende nichts anderes ergibt. Sofern das betreffende Material nicht unter der genannten Creative Commons Lizenz steht und die betreffende Handlung nicht nach gesetzlichen Vorschriften erlaubt ist, ist für die oben aufgeführten Weiterverwendungen des Materials die Einwilligung des jeweiligen Rechteinhabers einzuholen.

Weitere Details zur Lizenz entnehmen Sie bitte der Lizenzinformation auf http://creativecommons.org/ licenses/by/4.0/deed.de.

\section{Anhang}

Tab. 4 Übersicht Kategorien für die Dimensionen Agency und Communion

\begin{tabular}{ll}
\hline Agency & Lehrperson \\
5 & Die Lehrperson verhält sich sehr aktiv, \\
hoch & bestimmend und führt stark (z. B. befiehlt, \\
& fällt Schüler*innen ins Wort, schreit)
\end{tabular}

\section{Schülerinnen und Schüler}

Die Schülerinnen und Schüler verhalten sich sehr aktiv, bestimmend, versuchen das Unterrichtsgeschehen zu beeinflussen und zeigen viel Eigeninitiative (z. B. machen Vorschläge oder äussern Bemerkungen, widersprechen der Lehrperson)

Die Schülerinnen und Schüler verhalten sich aktiv und zeigen Eigeninitiative (z. B. fragen bei Unklarheiten nach, holen sich Unterstützung oder Materialien)

Die Schülerinnen und Schüler reagieren auf Aufforderungen der Lehrperson, zeigen jedoch wenig Eigeninitiative (z. B. erledigen Aufträge, beantworten Fragen, hören zu)

Die Schülerinnen und Schüler reagieren lediglich auf direkt an sie gerichtete Aufforderungen und zeigen keine Eigeninitiative (z. B. reagieren kaum, nur wenn sie explizit aufgerufen werden, sind abwartend, antworten leise oder ängstlich) 
Tab. 4 (Fortsetzung)

\begin{tabular}{|c|c|c|}
\hline $\begin{array}{l}1 \\
\text { niedrig }\end{array}$ & $\begin{array}{l}\text { Die Lehrperson verhält sich sehr passiv. } \\
\text { Sie zeigt kein Verhalten, mit dem das } \\
\text { Geschehen im Klassenzimmer beeinflusst } \\
\text { oder kontrolliert werden könnte (z.B. } \\
\text { interveniert nicht) }\end{array}$ & $\begin{array}{l}\text { Die Schülerinnen und Schüler verhalten sich } \\
\text { sehr passiv (z. B. beantworten Fragen nicht, } \\
\text { wirken verängstigt) }\end{array}$ \\
\hline $\begin{array}{l}\text { Commu- } \\
\text { nion }\end{array}$ & Lehrperson & Schülerinnen und Schüler \\
\hline $\begin{array}{l}5 \\
\text { hoch }\end{array}$ & $\begin{array}{l}\text { Die Lehrperson zeigt Wärme und ist } \\
\text { freundlich, jedoch distanzlos (z. B. wahrt } \\
\text { professionelle Distanz nicht, verhält sich } \\
\text { kumpelhaft, macht Sprüche oder Witze) }\end{array}$ & $\begin{array}{l}\text { Die Schülerinnen und Schüler sind herzlich } \\
\text { oder freundlich, jedoch kumpelhaft und di- } \\
\text { stanzlos gegenüber der Lehrperson (z. B. } \\
\text { umarmen oder berühren sie, verhalten sich } \\
\text { kumpelhaft) }\end{array}$ \\
\hline 4 & $\begin{array}{l}\text { Die Lehrperson zeigt Wärme und ist } \\
\text { freundlich und wahrt die professionelle } \\
\text { Distanz (z. B. lobt oder unterstützt Schü- } \\
\text { ler*innen, zeigt Wertschätzung, nimmt } \\
\text { sich Zeit) }\end{array}$ & $\begin{array}{l}\text { Die Schülerinnen und Schüler sind herzlich } \\
\text { oder freundlich, wahren aber eine angemes- } \\
\text { sene Distanz gegenüber der Lehrperson (z.B. } \\
\text { unterstützen die Lehrperson, freuen sich, } \\
\text { bedanken sich) }\end{array}$ \\
\hline 3 & $\begin{array}{l}\text { Die Lehrperson verhält sich neutral, sie } \\
\text { ist weder freundlich noch gereizt oder } \\
\text { unfreundlich (z.B. spricht in einem neu- } \\
\text { tralen Ton, reagiert oder antwortet recht } \\
\text { sachlich und kurz) }\end{array}$ & $\begin{array}{l}\text { Die Schülerinnen und Schüler sind neutral, } \\
\text { weder freundlich noch unfreundlich oder } \\
\text { gereizt gegenüber der Lehrperson (z.B. zei- } \\
\text { gen wenig positive oder negative Emotionen, } \\
\text { verhalten sich respektvoll) }\end{array}$ \\
\hline 2 & $\begin{array}{l}\text { Die Lehrperson ist kühl, unfreundlich } \\
\text { oder distanziert (z.B. reagiert gereizt, } \\
\text { unfreundlich, droht, unterbricht Schü- } \\
\text { ler*innen) }\end{array}$ & $\begin{array}{l}\text { Die Schülerinnen und Schüler wirken abwei- } \\
\text { send, reserviert, unfreundlich, provokativ oder } \\
\text { distanziert gegenüber der Lehrperson (z.B. } \\
\text { ignorieren die Lehrpersonen, machen sich } \\
\text { lustig, sind verängstigt, gehen wenig auf die } \\
\text { Lehrperson ein) }\end{array}$ \\
\hline $\begin{array}{l}1 \\
\text { niedrig }\end{array}$ & $\begin{array}{l}\text { Die Lehrperson ist feindselig gegenüber } \\
\text { den Lernenden (z. B. beschimpft oder } \\
\text { beleidigt oder wird handgreiflich) }\end{array}$ & $\begin{array}{l}\text { Die Schülerinnen und Schüler verhalten sich } \\
\text { feindselig gegenüber der Lehrperson (z. B. } \\
\text { verhalten sich aggressiv, widersetzen sich } \\
\text { aktiv, werden handgreiflich) }\end{array}$ \\
\hline
\end{tabular}

\section{Literatur}

Assor, A., Kaplan, H., Kanat-Maymon, Y., \& Roth, G. (2005). Directly controlling teacher behaviors as predictors of poor motivation and engagement in girls and boys: The role of anger and anxiety. Learning and Instruction, 15, 397-413.

Chang, M. (2009). An appraisal perspective of teacher burnout: examining the emotional work of teachers. Educational Psychology Review, 21, 193-218.

Davis, H. A. (2003). Conceptualizing the role and influence of student-teacher relationships on children's social and cognitive development. Educational Psychologist, 38, 207-234. https://doi.org/10.1207/ S15326985EP3804_2.

Deci, E.L., \& Ryan, R. M. (1985). Intrinsic motivation and self-determination in human behavior. New York: Plenum.

Eccles, J., Buchanan, C.M., Flanagan, C., Fuligni, A., Midgley, C., \& Yee, D. (1991). Control versus autonomy during early adolescence. Journal of Social Issues, 47(4), 53-68.

Einsiedler, W. (2017). Von Erziehungs- und Unterrichtsstilen zur Unterrichtsqualität. In M. K. W. Schweer (Hrsg.), Lehrer-Schüler-Interaktion: Inhaltsfelder, Forschungsperspektiven und methodische Zugänge (S. 267-287). Wiesbaden: Springer.

Friedman, I. A. (2006). Classroom management and teacher stress and burnout. In C. M. Evertson \& C. S. Weinstein (Hrsg.), Handbook of classroom management. Research, practice, and contemporary issues (S. 925-944). Mahwah: Lawrence Erlbaum. 
Granic, I., \& Hollenstein, T. (2003). Dynamic systems methods for models of developmental psychopathology. Development and Psychopathology, 15, 641-669. https://doi.org/10.1017/S0954579403000324.

Granic, I., \& Lamey, A. V. (2002). Combining dynamic systems and multivariate analyses to compare the mother-child interactions of externalizing subtypes. Journal of Abnormal Child Psychology, 30, 265-283.

Granic, I., Hollenstein, T., Dishion, T. J., \& Patterson, G. R. (2003). Longitudinal analysis of flexibility and reorganization in early adolescence: a dynamic systems study of family interactions. Developmental Psychology, 39, 606-617.

Granic, I., O’Hara, A., Pepler, D., \& Lewis, M.D. (2007). A dynamic systems analysis of parent-child changes associated with successful "real-world" interventions for aggressive children. Journal of Abnormal Child Psychology, 35, 845-857. https://doi.org/10.1007/s10802-007-9133-4.

Gurtman, M. B. (2009). Exploring personality with the interpersonal circumplex. Social and Personality Psychology Compass, 3, 601-619. https://doi.org/10.1111/j.1751-9004.2009.00172.x.

Herzog, W. (2009). Schule und Schulklasse als soziale Systeme. In R. Becker (Hrsg.), Lehrbuch der Bildungssoziologie (S. 155-194). Wiesbaden: VS.

Hinde, R. A. (1976a). Interactions, relationships and social structure. Man, 11(1), 1-17.

Hinde, R. A. (1976b). On describing relationships. Journal of Child Psychology and Psychiatry, 17(1), $1-19$.

Hinde, R. A. (1997). Relationships: a dialectical perspectiv. Hove: Psychology Press.

Hinde, R. A., \& Tajfel, H. (1979). Towards understanding relationships. Bd. 18. London: Academic Press.

Hollenstein, T. (2007). State space grids: Analyzing dynamics across development. International Journal of Behavioral Development, 31, 384-396. https://doi.org/10.1177/0165025407077765.

Hollenstein, T. (2013). State space grids: depicting dynamics across development. Boston: Springer US.

Hollenstein, T., Granic, I., Stoolmiller, M., \& Snyder, J. (2004). Rigidity in parent-child interactions and the development of externalizing and internalizing behavior in early childhood. Journal of Abnormal Child Psychology, 32(6), 595-607.

Kelle, U., \& Kluge, S. (2010). Vom Einzelfall zum Typus. Fallvergleiche und Fallkontrastierung in der qualitativen Sozialforschung. Wiesbaden: VS.

Kenrick, D. T., \& Funder, D.C. (1988). Profiting from controversy: Lessons from the person-situation debate. American Psychologist, 43, 23-34.

Klieme, E., Lipowsky, F., Rakoczy, K., \& Ratzka, N. (2006). Qualitätsdimensionen und Wirksamkeit von Mathematikunterricht. In M. Prenzel \& L. Allolio-Näcke (Hrsg.), Untersuchungen zur Bildungsqualität von Schule (S. 127-146). Münster: Waxmann.

Koopmans, M., \& Stamovlasis, D. (2016). Introduction to education as a complex dynamical system. In M. Koopmans \& D. Stamovlasis (Hrsg.), Complex dynamical systems in education: concepts, methods and applications (S. 1-8). Cham: Springer.

Kunter, M., \& Ewald, S. (2016). Bedingungen und Effekte von Unterricht: Aktuelle Forschungsperspektiven aus der pädagogischen Psychologie. In N. McElvany, W. Bos, H. G. Holtappels, M. M. Gebauer \& F. Schwabe (Hrsg.), Bedingungen und Effekte guten Unterrichts (S. 9-31). Münster: Waxmann.

Lamey, A., Hollenstein, T., Lewis, M. D., \& Granic, I. (2004). GridWare (Version 1.1). [Computer software]. http://statespacegrids.org. Letzter Zugriff: 04.11.2020

Mainhard, M. T., Brekelmans, M., \& Wubbels, T. (2011). Coercive and supportive teacher behaviour: within- and across-lesson associations with the classroom social climate. Learning and Instruction, 21, 345-354. https://doi.org/10.1016/j.learninstruc.2010.03.003.

Mainhard, M. T., Pennings, H.J.M., Wubbels, T., \& Brekelmans, M. (2012). Mapping control and affiliation in teacher-student interaction with State Space Grids. Teaching and Teacher Education, 28, 1027-1037. https://doi.org/10.1016/j.tate.2012.04.008.

Martin, C.L., Fabes, R.A., Hanish, L.D., \& Hollenstein, T. (2005). Social dynamics in the preschool. Developmental Review, 25, 299-327.

Obsuth, I., Murray, A. L., Malti, T., Sulger, P., Ribeaud, D., \& Eisner, M. (2017). A non-bipartite propensity score analysis of the effects of teacher-student relationships on adolescent problem and prosocial behavior. Journal of Youth and Adolescence, 46, 1661-1687. https://doi.org/10.1007/s10964-0160534-y.

Ophardt, D., \& Thiel, F. (2017). Klassenmanagement als Basisdimension der Unterrichtsqualität. In M. K.W. Schweer (Hrsg.), Lehrer-Schüler-Interaktion: Inhaltsfelder, Forschungsperspektiven und methodische Zugänge (S. 245-266). Wiesbaden: Springer.

Park, S., Holloway, S. D., Arendtsz, A., Bempechat, J., \& Li, J. (2012). What makes students engaged in learning? A time-use study of within- and between-individual predictors of emotional engagement 
in low-performing high schools. Journal of Youth and Adolescence, 41, 390-401. https://doi.org/10. 1007/s10964-011-9738-3.

Pennings, H. J. M., \& Hollenstein, T. (2019). Teacher-student interactions and teacher interpersonal styles: a state space grid analysis. The Journal of Experimental Education. https://doi.org/10.1080/ 00220973.2019 .1578724 .

Pennings, H. J. M., \& Mainhard, T. (2016). Analyzing teacher-student interactions with State Space Grids. In M. Koopmans \& D. Stamovlasis (Hrsg.), Complex dynamical systems in education: Concepts, methods and applications (S. 233-271). Cham: Springer. https://doi.org/10.1007/978-3-319-27577$2 \_12$.

Pennings, H. J. M., Brekelmans, M., Sadler, P., Claessens, L.C. A., van der Want, A. C., \& van Tartwijk, J. (2018). Interpersonal adaptation in teacher-student interaction. Learning and Instruction, 55, 41-57. https://doi.org/10.1016/j.learninstruc.2017.09.005.

Pennings, H.J.M., Brekelmans, M., Wubbels, T., van der Want, A.C., Claessens, L.C.A., \& van Tartwijk, J. (2014a). A nonlinear dynamical systems approach to real-time teacher behavior: differences between teachers. Nonlinear Dynamicsm Psychology, and Life Sciences, 18, 23-45.

Pennings, H.J.M., van Tartwijk, J., Wubbels, T., Claessens, L.C.A., van der Want, A.C., \& Brekelmans, M. (2014b). Real-time teacher-student interactions: A Dynamic Systems approach. Teaching and Teacher Education, 37, 183-193. https://doi.org/10.1016/j.tate.2013.07.016.

Pianta, R. C. (2006). Classroom management and relationships between children and teachers: implications for research and practice. In C. M. Evertson \& C. S. Weinstein (Hrsg.), Handbook of classroom management: Research, practice and contemporary issues (S. 685-709). Mahwah: Lawrence Erlbaum.

Pianta, R.C., \& Hamre, B.K. (2009). Conceptualization, measurement, and improvement of classroom processes: standardized observation can leverage capacity. Educational Researcher, 38(2), 109-119.

Pianta, R.C., Hamre, B., \& Stuhlman, M. (2003). Relationships between teachers and children. In W. M. Reynolds \& G. E. Miller (Hrsg.), Educational psychology. Handbook of Psychology, (Bd. 7, S. 199-234). Hoboken: Wiley \& Sons.

Praetorius, A.-K., Klieme, E., Herbert, B., \& Pinger, P. (2018). Generic dimensions of teaching quality: the German framework of three basic dimensions. ZDM, 50(3), 407-426. https://doi.org/10.1007/ s11858-018-0918-4.

Scherzinger, M., Wettstein, A., \& Wyler, S. (2018). Aggressive und nicht aggressive Unterrichtsstörungen durch Schüler*innen sowie durch Klassen- und Fachlehrpersonen - eine Videostudie. Empirische Sonderpädagogik, 4, 388-407.

Spilt, J. M., Koomen, M. Y., \& Thijs, J.T. (2011). Teacher wellbeing: the importance of teacher-student relationships. Educational Psychology Review, 23, 457-477.

Suldo, S., Friedrich, A., White, T., Farmer, J., Devon, M., \& Michalowski, J. (2009). Teacher support and adolescents' subjective well-being: a mixed-methods investigation. School Psychology Review, 38, 67-85.

Tausch, A.-M., \& Tausch, R. (1973). Erziehungspsychologie. Göttingen: Hogrefe.

Thies, B. (2017). Forschungszugänge zur Lehrer-Schüler-Interaktion: Ein historischer Abriss. In M. K.W. Schweer (Hrsg.), Lehrer-Schüler-Interaktion: Inhaltsfelder, Forschungsperspektiven und methodische Zugänge (S. 65-88). Wiesbaden: Springer.

Van Geert, P. (2009). Nonlinear complex dynamical systems in developmental psychology. In S. J. Guastello, M. Koopmans \& D. Pincus (Hrsg.), Chaos and complexity in psychology: the theory of nonlinear dynamical systems (S. 242-281). New York: Cambridge University Press.

Wentzel, K. R. (2002). Are effective teachers like good parents? Teaching styles and student adjustment in early adolescence. Child Development, 73(1), 287-301.

Wentzel, K. R. (2010). Students' relationships with teachers. In J. L. Meece \& J. S. Eccles (Hrsg.), Handbook of research on schools, schooling, and human development (S. 75-91). New York: Routledge.

Wentzel, K. R. (2012). Teacher-student relationships and adolescent competence at school. In T. Wubbels, P. den Brok, J. van Tartwijk \& J. Levy (Hrsg.), Advances in learning environments research: volume 3. Interpersonal relationships in education: an overview of contemporary research (S. 19-36). Rotterdam, Boston, Taipei: Sense Publishers.

Wettstein, A. (2013). Die Wahrnehmung sozialer Prozesse im Unterricht. Schweizerische Zeitschrift für Heilpädagogik, 7/8, 5-13.

Wettstein, A., \& Scherzinger, M. (2019). Unterrichtsstörungen verstehen und wirksam vorbeugen. Stuttgart: Kohlhammer.

Wettstein, A., Ramseier, E., \& Scherzinger, M. (2018). Eine Mehrebenenanalyse zur Schülerwahrnehmung von Störungen im Unterricht der Klassen- und einer Fachlehrperson. Psychologie in Erziehung und Unterricht, 65(1), 1-16. 
Wettstein, A, Ramseier, E., Scherzinger, M., \& Gasser, L. (2016). Unterrichtsstörungen aus Lehrerund Schülersicht. Zeitschrift für Entwicklungspsychologie und Pädagogische Psychologie, 48(4), 171-183.

Wettstein, A., Scherzinger, M., \& Ramseier, E. (2018). Empirische Arbeit: Unterrichtsstörungen, Beziehung und Klassenführung aus Lehrer-, Schüler- und Beobachterperspektive. Psychologie in Erziehung und Unterricht, 65(1), 58-74.

Wettstein, A., Scherzinger, M., \& Wyler, S. (2016). Fragebogen zur Erfassung von Störungen im Unterricht: die Faktorstruktur der Schülerinnen- und Schülerversion. Empirische Sonderpädagogik, 2, 189-202.

Wubbels, T., \& Brekelmans, M. (2005). Two decades of research on teacher-student relationships in class. International Journal of Educational Research, 43, 6-24. https://doi.org/10.1016/j.ijer.2006.03.003.

Wubbels, T., Brekelmans, M., den Brok, P., \& van Tartwijk, J. (2006). An interpersonal perspective on classroom management in secondary classrooms in the Netherlands. In C. M. Evertson \& C.S. Weinstein (Hrsg.), Handbook of classroom management: research, practice and contemporary issues (S. 1161-1191). Mahwah: Lawrence Erlbaum.

Wubbels, T., Brekelmans, M., den Brok, P., Wijsman, L., Mainhard, T., \& van Tartwijk, J. (2015). Teacherstudent relationships and classroom management. In E. T. Emmer \& E. J. Sabornie (Hrsg.), Handbook of classroom management (2. Aufl. S. 363-386). New York: Routledge.

Wubbels, T., Brekelmans, M., Mainhard, M.T., den Brok, P., \& van Tartwijk, J. (2016). Teacher-student relationships and student achievement. In K. R. Wentzel \& G. B. Ramini (Hrsg.), Handbook of social influences in school contexts: social-emotional, motivation, and cognitive outcomes. Educational Psychology Handbook. (S. 127-143). New York: Routledge. 